\title{
Content based Image Retrieval using Combined Features
}

\author{
Darshan Ingle \\ Lecturer, Comp Engg Dept, \\ Thadomal Shahani Eng College \\ Bandra, Mumbai.
}

\author{
Shalini Bhatia \\ Asst Prof, Comp Engg Dept, \\ Thadomal Shahani Eng College \\ Bandra, Mumbai.
}

\begin{abstract}
Content Based Image Retrieval is the application of computer vision techniques to the image retrieval problem of searching for digital images in large databases. The method of CBIR discussed in this paper can filter images based their content and would provide a better indexing and return more accurate results. In this paper techniques to retrieve image based on their content namely color, texture and combined retrieval using both the features together. Combined feature retrieval gives better and more accurate result as compared to using a single technique for retrieval.
\end{abstract}

\section{General Terms}

Colour histogram, GLCM, Combined colour and texture features, Euclidean distance.

\section{Keywords}

Precision, Recall, Combined-feature, Precision-Recall Cross over point.

\section{INTRODUCTION}

Content-based image retrieval (CBIR), also known as query by image content (QBIC) is the application of computer vision techniques to the image retrieval problem, that is, the problem of searching for digital images in large databases. "Content-based" means that the search will analyze the actual contents of the image rather than the metadata such as keywords, tags, and/or descriptions associated with the image. The term 'content' in this context might refer to colors, shapes, textures, or any other information that can be derived from the image itself. Thus a system that can filter images based on their content would provide better indexing and return more accurate results [1].

\section{DESIGN CONSIDERATIONS:}

\subsection{Color feature extraction}

In color feature extraction, the histogram distance for both the query image and first image in the database is calculated i.e contribution of RGB components is calculated individually. Now the Euclidean distance between the query image and the first image in the database is calculated. This Euclidean distance is stored in an array. The procedure is repeated for the remaining images in the database followed by storing their respective values. Now the array is sorted in ascending order and the first 8 closest matches are displayed.

\subsection{Texture feature extraction}

In texture feature extraction, the texture feature vector is extracted using the grey level co-occurrence matrix[3] for the query image and the first image in the database. The co- occurrence matrix representation is one such technique which gives us not only the intensity values but also the distribution of the intensities. The features selected for retrieving texture properties are Energy, Contrast, Entropy, Inverse difference, Moment of inertia, Mean, Variance, Skewness, Distribution uniformity, Local stationary and Homogeneity.

Now the Euclidean distance between the query image and the first image in the database is calculated. This Euclidean distance is stored in an array. The procedure is repeated for the remaining images in the database followed by storing their respective values. Now the array is sorted in ascending order and the first 8 closest matches are displayed.

\subsection{Combined feature extraction [6]}

In combined feature extraction, the histogram distance for both the query image and first image in the database is calculated. Now the Euclidean distance between the query image and the first image in the database is calculated. This Euclidean distance is stored in an array. The procedure is repeated for the remaining images in the database followed by storing their respective values.

Now, the texture feature vector is extracted using the grey level co-occurrence matrix for the query image and the first image in the database. The Euclidean distance between the query image and the first image in the database is calculated. This Euclidean distance is stored in an array. The procedure is repeated for the remaining images in the database followed by storing their respective values.

The above sorted values are now used for the further calculations. Euclidean distance in between the above calculated arrays is calculated. These Euclidean distances are now sorted in ascending order and the first 8 closest matches are displayed. 


\section{ALGORITHM}

\subsection{Algorithm for Color feature extraction}

1. Query image is given as input to the system.

2. Calculate the histogram distance for the query image by histogram method

3. Calculate the histogram distance for the first image in the database.

4. Calculate the Euclidean distance between the query image and the image in the database.

Images are extracted from the database using colour feature by Euclidean distance algorithm.

5. Store this distance in an array 'dist'.

6. Calculate the histogram distance for the next image in the database.

7. Repeat steps 4,5 and 6 till the last image in the database is reached.

8. After this, sort the distance array showing the Euclidean distances in ascending order.

9. Display first 8 results as the closest matches.

\subsection{Algorithm for texture feature \\ extraction}

1. Query image is given as input to the system.

2. Calculate the texture feature vector for the query image using the grey-level co-occurrence matrix i.e GLCM and store it in an array.

3. Calculate the texture feature vector for the first image in the database using the grey-level cooccurrence matrix and store it in an array.

4. Calculate the Euclidean distance between the query image and the image in the database.

5. Store this in an array 'dist'.

6. Calculate the texture feature vector for the next image in the database using the grey-level cooccurrence matrix and store it in an array.

7. Repeat steps 4,5 and 6 till the last image in the database is reached.

8. After this, sort the distance array showing the Euclidean distances in ascending order.

9. Display first 8 results as the closest matches

\subsection{Algorithm for Combined feature extraction (color and texture both)}

1. Query image is given as input to the system.

2. Calculate the histogram distance for the query image by histogram method

3. Calculate the histogram distance for the first image in the database.

4. Calculate the Euclidean distance between the query image and the image in the database.

5. Store this distance in an array 'dist'.

6. Calculate the histogram distance for the next image in the database.

7. Repeat steps 4,5 and 6 till the last image in the database is reached.

8. After this, sort the distance array 'dist' showing the Euclidean distances in ascending order.

9. Calculate the texture feature vector for the query image using the grey-level co-occurrence matrix.

10. Calculate the texture feature vector for the first image in the database using the grey-level cooccurrence matrix and store it in an array.

11. Calculate the Euclidean distance between the query image and the image in the database.

12. Store this in an array 'dist1'.
13. Calculate the texture feature vector for the next image in the database using the grey-level cooccurrence matrix and store it in an array.

14. Repeat steps 11,12 and 13 till the last image in the database is reached.

15. After this, sort the distance array 'distl' showing the Euclidean distances in ascending order.

16. Now calculate the Euclidean distance between the array 'dist' and 'dist1'.

17. Integrate the above results to get an effective search retrieval result by displaying the first 8 results as the closest matches.

\section{RESULTS}

\subsection{Color Histogram method}

Following results are achieved for color feature extraction.

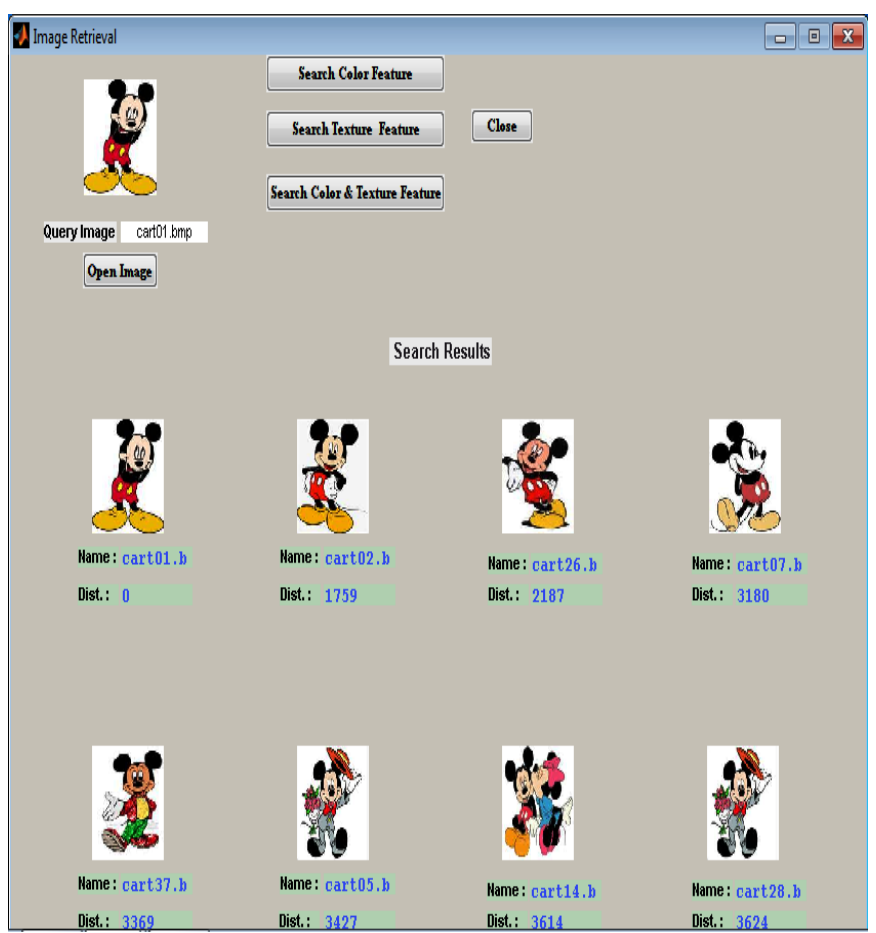

Fig 1: Results of Color feature for general database 


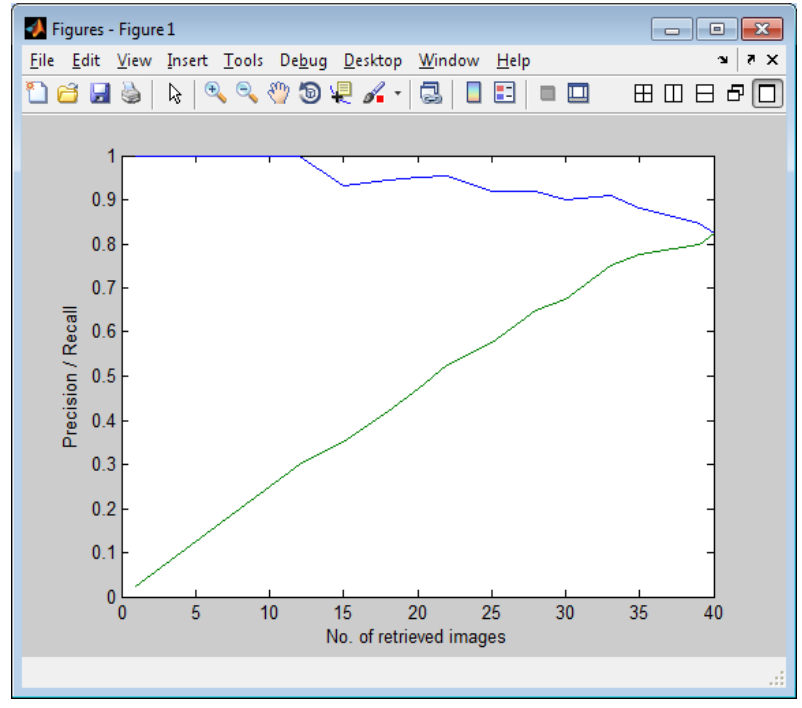

Fig 2: Calculation of COP for Color feature

Crossover point $=\mathbf{0 . 8 2 5}$

\subsection{Results of GLCM method for general database}

Following results are achieved for texture feature extraction.

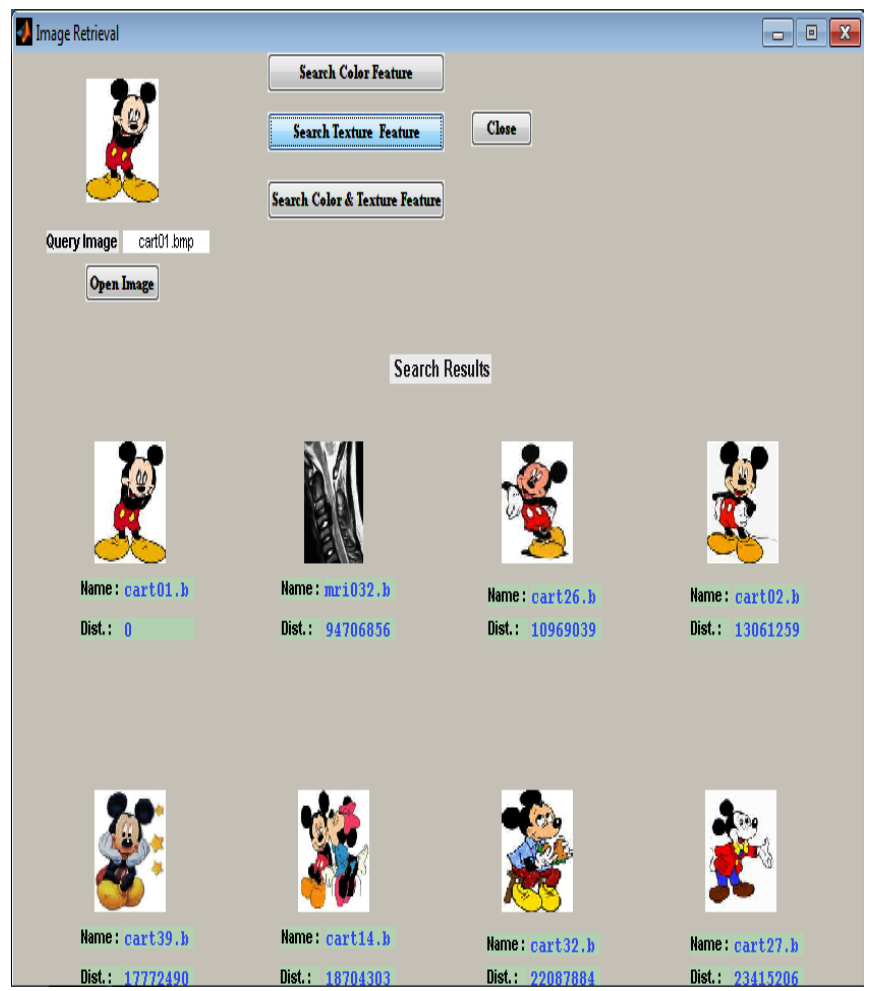

Fig 3: Results of Texture feature for general database

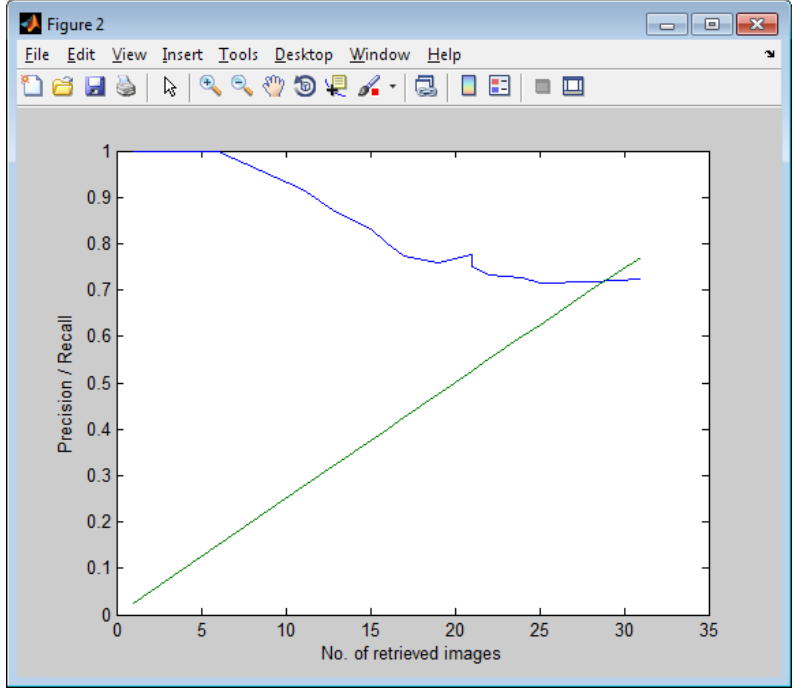

Fig 4: Calculation of COP for Texture feature

Crossover point $=\mathbf{0 . 7 7 7}$

\subsection{Results of Combined feature method for general database}

Following results are achieved for combined feature extraction.

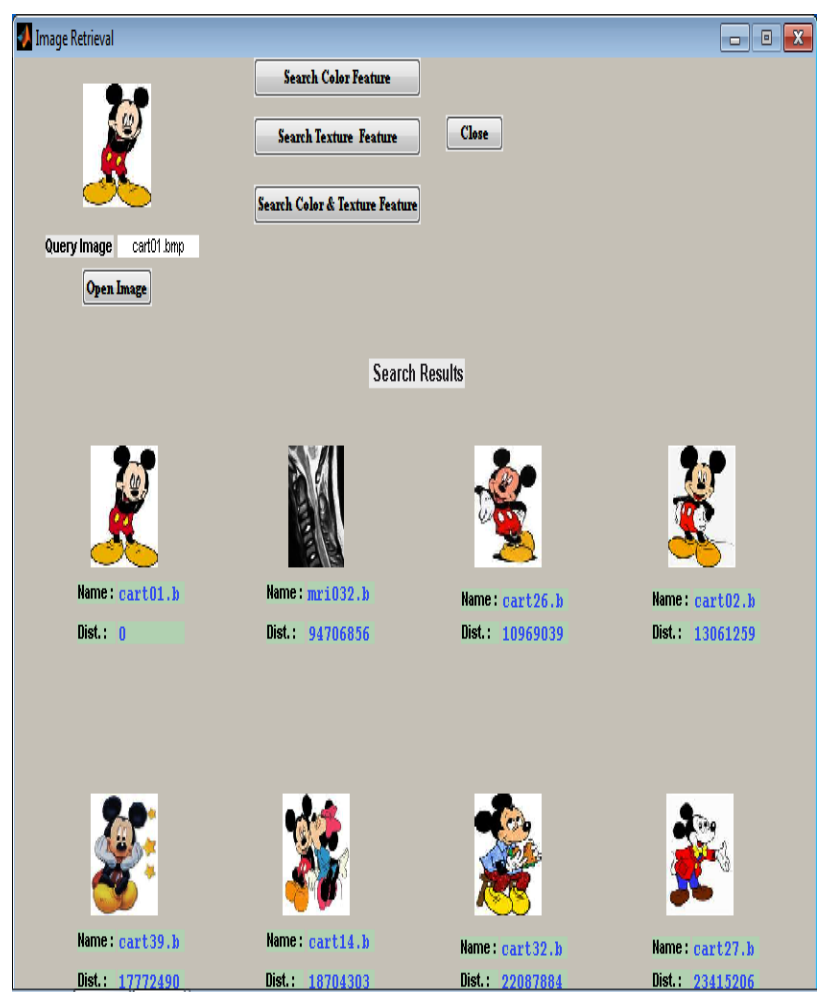

Fig 5: Results of Combined feature for general database 


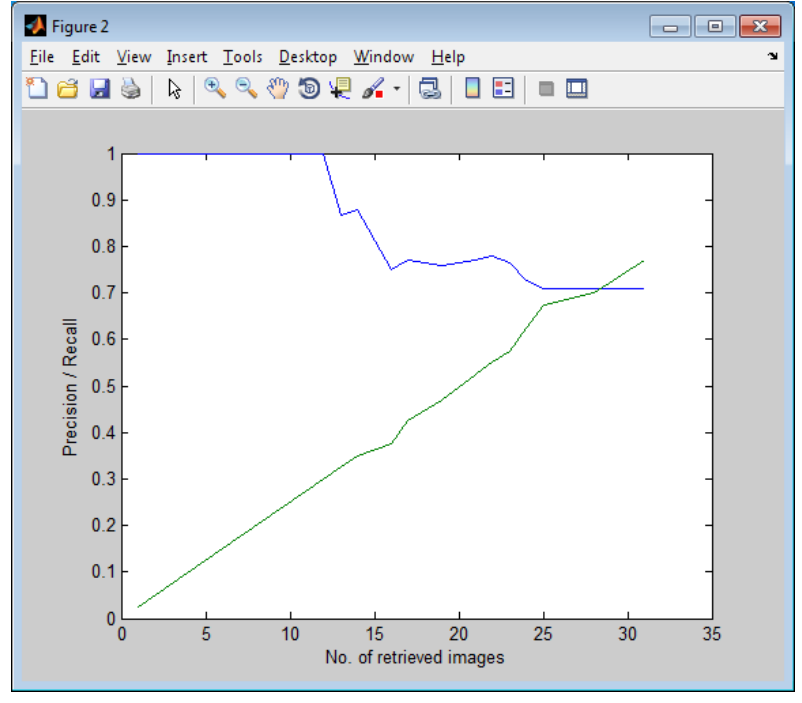

Fig 6: Calculation of COP for Colour+Texture feature

Crossover point $=0.7$

\section{CONCLUSION}

Colour, texture and combined features are implemented for various codebook sizes on general database.

For colour feature extraction, colour histogram method is implemented.

For texture, in all eleven feature which includes energy, contrast, entropy, inverse difference, mean, variance, distribution uniformity, moment of inertia, skewness, local stationary, homogeneity are used. These features together increase the retrieval efficiency of the texture feature.

For combined feature extraction, we extract the color and texture features altogether i.e all the above incorporated features and get a combined result retrieved.

The result of retrieval varies upon the content type of image. Thus, successful implementation of the feature extraction is achieved for the project.

\section{FURTHER WORK}

The Content based image retrieval systems can be enhanced in many ways and there will always be new developments in this technology and new areas for researchers to explore. The following will briefly present further enhancements of isolated word speech recognition system.

The system shall be made to accept images that are invariant to rotation. In other words, this system shall be improved further extract even those images that are rotated and would have been missed if they are not aligned in properly as required. The system shall be made to accept images that are invariant to translation. The system shall be made to accept images that are invariant to scaling. Morphological features of the image can also be considered as one of the feature vector for extracting the shape characteristics.

The system can be further made efficient enough for the user so that the query image can be searched in the database by choosing the desired feature vector or a combination of feature vectors.

\section{ACKNOWLEDGMENTS}

The work presented in this paper was completed as a part of M.E. course in Computer Engineering, Mumbai University. I have great pleasure in presenting this paper on "CONTENT BASED IMAGE RETRIEVAL USING COMBINED FEATURES". I take opportunity to thank all those who have contributed in successful completion of this report.

I wish to express sincere thanks and deep sense of gratitude to respected mentor and guide Ms. Shalini Bhatia, Assistant Professor, Computer Engineering Department for her in depth and enlightening support with all of her kindness. Apart from technical advice I have got lots of encouragement, which motivates to strive harder for excellence. Despite her busy schedule she was always kind and patient to listen to my difficulties and indicate to me the right path.

I am also thankful to Mr. Jayant Gadge, H.O.D., Computer Engineering Department for his continuous support and encouragement to achieve always the best in whatsoever I am doing.

A word of thanks is also due to my parents who had given me life and taught me what is to be done with it.

\section{REFERENCES}

[1] Woo Chaw Seng, Seyed Hadi Mirisaee, "A Content-Based Retrieval System for Blood Cells Images", International Conference on Future Computer and Communication, icfcc, pp.412-415, 2009.

[2] Mohammad Reza Zare, Raja Noor Ainon, Woo Chaw Seng, "Content-Based Image Retrieval for Blood Cells" ,Third Asia International Conference on Modelling \& Simulation, ams, pp.332-335, 2009.

[3] Fan-Hui Kong, "Image Retrieval Using Both Color And Texture Features", Department of Information Science \& Technology, Heilongjiang Proceedings of the Eighth International Conference on Machine Learning and Cybernetics, Baoding, 12-15 July 2009.

[4] Xiangbin Wang, Junmin He, Zhongwei Lv , "Texturebased Retrieval of Thyroid Gland SPECT Image", School of Life Science and Technology Tongji University Shanghai, China, 978-1-4244-41341/09/\$25.00 @2009 IEEE.

[5] Subrahmanyam Murala, Anil Balaji Gonde, R. P. Maheshwari, "Color and Texture Features for Image Indexing and Retrieval", Department of Electrical Engineering, Indian Institute of Technology Roorkee,Uttarakhand, India, IEEE International Advance Computing Conference @2009.

[6] Wei Wang, Motoyuki Suzuki "Texture Retrieval Based on Gray-Primitive Cooccurrence Matrix", Dept InfoScience \& Intelligent Systems Faculty Engineering, , The University of kushima, Tokushima, Japan, 978-1-42446899-7/10/\$26.00 @2010 IEEE. 